\title{
IrCR Thrombosis
}

National Cancer Institute

\section{Source}

National Cancer Institute, irCR Thrombosis. NCI Thesaurus. Code C147910.

Complete resolution of thrombosis, with recanalization of vessel. 